$\begin{array}{cl}\begin{array}{c}\text { Revue } \\ \text { de Ihistoire } \\ \text { des religions }\end{array} & \text { Revue de l'histoire des religions } \\ & \begin{array}{l}1 \mid 2010 \\ \text { Beauté du rite }\end{array}\end{array}$

\title{
Le Signe du Vendredi Saint
}

L'esthétique liturgique selon Letourneux

The Sign of Holy Friday: Liturgical Esthetics According to Letourneux

\section{Christian Belin}

\section{CpenEdition}

Journals

Édition électronique

URL : http://journals.openedition.org/rhr/7559

DOI : $10.4000 /$ rhr.7559

ISSN : 2105-2573

Éditeur

Armand Colin

Édition imprimée

Date de publication : 1 mars 2010

Pagination : 33-53

ISBN : 978-2200-92612-0

ISSN : 0035-1423

Référence électronique

Christian Belin, « Le Signe du Vendredi Saint », Revue de l'histoire des religions [En ligne], 1 | 2010, mis en ligne le 01 mars 2013, consulté le 19 avril 2019. URL : http://journals.openedition.org/rhr/7559; DOI : 10.4000/rhr.7559 


\section{Le Signe du Vendredi Saint L'esthétique liturgique selon Letourneux}

Apôtre de la liturgie, Letourneux souhaitait revitaliser le lien émotionnel qui relie le fidèle à toute liturgie. Par des commentaires destinés à " inspirer » le « sentiment » des chrétiens, il scrute un mystère sémiotique déployé dans le temps liturgique. Il en contemple la disposition, dans l'esprit de cette esthétique théologique analysée par Urs von Balthasar. Cette étude privilégie la liturgie singulière du Vendredi Saint, rebelle a priori à toute approche esthétique, puisqu'en ce jour le dépouillement maximal des modes d'expression mime une véritable dramaturgie de la Passion. Le sentiment religieux, confondu avec la mystagogie, consacre la beauté paradoxale du Serviteur défiguré, en cette Gloire enténébrée où se consomment la mort et la résurrection du Signe, en quelque sorte la pâque de toute esthétique chrétienne.

\section{The Sign of Holy Friday : \\ Liturgical Esthetics According to Letourneux}

An apostle of the liturgy, Letourneux wished to revitalize the emotional link between the faithful and the liturgy. Through commentaries destined to inspire the sentiments of Christians, he scrutinizes a semiotic mystery that unfolds within the liturgical time frame. In the spirit of theological esthetics as analyzed by Urs von Balthazar, he studies its disposition of this mystery. The present study focuses on the Good Friday liturgy, seemingly irrelevant to any aesthetic approach, since the extreme sobriety of expressive modes on this day is the enactment of a veritable dramaturgy of the Passion of Christ. Religious sentiment, merging with mystagogy, consacrates the paradoxical beauty of the marred Servant, in a dimmed glory where the death and resurrection of the Sign, as it were the Passover of all Christian esthetics, are acted out. 
Le mot « liturgie » ne s'est vraiment imposé, en langue vulgaire, qu'aux $\mathrm{XVI}^{\mathrm{e}}$ et $\mathrm{XVII}{ }^{\mathrm{e}}$ siècles, pour désigner l'ensemble des cérémonies qui régissent le culte public rendu à Dieu, dans l'Église. Même si le mot grec leitourgia est utilisé dans la Septante pour traduire l'hébreu aboda (service du Temple), et pareillement dans le Nouveau Testament (où le Christ est désigné comme le liturge par excellencel), le mot liturgie n'est tombé dans l'usage courant qu'à partir du concile de Trente, quand l'Église a renouvelé sa propre réflexion sur les liens qui unissent le dogme à ses expressions cultuelles. Sans doute aussi, en cette période de controverse anti-réformée, l'Église éprouvait-elle le désir de mieux enraciner dans le vocabulaire des Écritures le riche patrimoine littéraire et artistique de ses cérémonies. $\mathrm{Au} \mathrm{XVII}{ }^{\mathrm{e}}$ siècle en particulier se sont multipliées les études sur l'histoire, la nature et le sens de la liturgie chrétienne ${ }^{2}$. Henri Bremond a pu parler d'une "inquiétude liturgique», caractéristique de ce siècle, et voir par exemple dans L'Année chrétienne de Nicolas Letourneux un véritable « catéchisme liturgique ${ }^{3}$ ».

Le projet de Letourneux ${ }^{4}$ ne consistait, d'abord, ni à offrir un commentaire des textes bibliques insérés dans le cycle liturgique,

1. Le Christ est appelé «ministre du sanctuaire (tôn agiôn leitourgos)» dans Hébreux, VIII, 2. Autres emplois du mot dans Luc, I, 23; Philippiens, II, 17; Romains, XV, 16 (Paul se désigne comme le desservant, leitourgos, du Christ).

2. Citons en particulier, au sein de la vaillante congrégation de Saint-Maur, le bénédictin Jean Mabillon (1632-1707) qui écrivit un De liturgia gallicana, 1685, et le bénédictin Edmé Martène (1654-1739) pour ses deux volumineux ouvrages, De antiquis monachorum ritibus (2 vol., 1690) et De antiquis Ecclesiae ritibus (4 vol., 1700-1738).

3. Histoire littéraire du sentiment religieux en France, tome X, Paris, Bloud et Gay, 1932, respectivement p. 34 et 51. Dans ce même volume X, La Prière et les prières de l'Ancien régime, Bremond parle encore de la « hantise liturgique du classicisme dévot », p. 214. Le chapitre 2 de ce volume X est consacré à Letourneux et à la propagande liturgique, p. 33-58.

4. Nicolas Letourneux (1640-1686), ordonné prêtre en 1662, a publié dès 1673 un Office de la semaine sainte, latin et français. Le premier volume de l'Année chrétienne fut publié en 1681 , les autres entre 1682 et 1685 . Après la mort de Letourneux, Ernest Ruth d'Ans rédigera les tomes X et XI. Un an après sa mort, en 1687, paraît également le Bréviaire romain en latin et en français. L'Année chrétienne sera mise à l'Index en 1695, en raison de sa traduction de l'Ordinaire de la messe. Letourneux fut également un prédicateur qui enthousiasmait la Cour (entre autres Madame de Sévigné) par la simplicité évangélique de ses sermons. Sur la biographie de Letourneux, ami de Port-Royal, voir Sainte-Beuve, Port-Royal, 
ni à se montrer attentif aux dimensions extérieures du culte, au spectacle cérémoniel, ni même encore à promouvoir une meilleure appréhension rationnelle de la doctrine ainsi traduite par des gestes ou des symboles. Les trois démarches n'étaient pas séparables à ses yeux, et l'Année chrétienne louvoie en quelque sorte entre ces différents modes de perception ou de connaissance, pour se placer à mi-chemin entre le sensible et l'intelligible, justement parce que la liturgie orchestre un mystère de foi à travers une médiation matérielle qui sollicite la sensation, le sentiment, l'aisthesis.

Inaugurant son œuvre monumentale, Letourneux affiche clairement son intention : « Ce livre pourra servir à leur inspirer ces sentiments par l'explication qu'il contient des prières et les lectures ${ }^{5}$. » L'ouvrage veut contribuer à l'éducation des sens (physiques et spirituels), désormais mis au service du Sens, de la révélation chrétienne, dont un commentaire interroge la disposition temporelle au sein du cycle liturgique. Si la liturgie signifie d'abord, en effet, le lieu où se révèle la Gloire divine (beauté ineffable), elle devient aussi, sur un mode responsorial, le lieu où s'expriment les « sentiments » du peuple fidèle : motions intérieures, émotions diffuses qui combinent pensées et sensations. Letourneux insiste sur la nécessité d'une telle « réponse » en recommandant de ne pas « aller les mains vides au-devant de ce Roi qui vient $»^{6}$. Le déploiement éternel du mystère dans l'économie salvifique se répète dans le circuit temporel de la liturgie. À travers les cérémonies, perçues dans leur totalité harmonieuse (textes, paroles, musique, gestes, objets, etc.), la Gloire divine est à la fois révélée et révélante, source et objet de révélation, cause finale de n'importe quel signe liturgique. Lorsqu'il commente l'année chrétienne, Letourneux ne fait donc que contempler la disposition liturgique des offices, cherchant à pénétrer plus avant dans la communication humano-divine qui la traverse et qui demeure toujours une communion, une synergie, un admirabile commercium.

Paris, Hachette, 1912, tome cinquième, p. 210-234. Bremond reprend largement les informations données par Sainte-Beuve. Édition utilisée : L'Année chrétienne, contenant les messes des dimanches, fêtes et féries de toute l'année, en latin et en français, avec explication des Épitres et des Évangiles, Paris, Charles Robustel, Jean-François Josse, Charles Jean-Baptiste Delespine, 1736.

5. Année chrétienne..., op. cit., t. I, p. 4.

6. Ibid. 
Que l'on donne au mot "esthétique » son sens étymologique (sensation, sentiment) ou qu'on le considère dans son acception post-kantienne ${ }^{7}$ (jugement ou appréciation portant sur la nature du Beau), il est indéniable que la dimension esthétique est constitutive de la liturgie chrétienne. Bien avant la naissance philosophique du concept d'esthétique, l'Église a toujours su que ses cérémonies devaient emprunter aux langages artistiques leurs modes d'expression spécifiques pour concourir à la manifestation d'une Vérité dont la beauté n'a d'égale que sa propre transcendance ${ }^{8}$. Dans l'étude magistrale consacrée à "l'esthétique théologique », Hans Urs von Balthasar a particulièrement insisté sur cette « esthétique » d'avant l'esthétique, dont on peut suivre l'histoire depuis l'époque des Pères ${ }^{9}$; il reconnaissait aussi volontiers les difficultés d'utilisation de ce concept, toujours soupçonné de contamination profane ou de velléité hégémonique, surtout lorsqu'il prétend absorber l'élément théologique. Le croisement périlleux des compétences peut en effet conduire à esthétiser la théologie, alors qu'il importerait de concevoir l'esthétique à l'intérieur d'un discours théologique qui

7. Hans Urs von Balthasar analyse la valeur polémique du terme «esthétique » dans la théologie libérale réformée, après Hegel et Kirkegaard : La Gloire et la Croix. Les aspects esthétiques de la Révélation, t. I Apparition, traduit de l'allemand par Robert Givord, Paris, Aubier, 1965 (Herrlichkeit. Eine theologische Âsthetik. I Schau der Gestalt, Einsiedeln, Johannes Verlag, 1961), en particulier p. 42-43.

8. Dans son Esthétique, Hegel reconnaît que « pour être un contenu authentique de l'art, la vérité doit avoir pour détermination même de pouvoir émerger dans le sensible ", Esthétique, trad. Charles Bénard, Paris, Livre de poche, 1995, p. 60. Mais on sait qu'il perçoit cette émergence essentiellement dans l'art classique grec. Il écrit par exemple : «C'est parce que le christianisme porte à la représentation Dieu en tant qu'esprit, non pas en tant qu'esprit individuel particulier, mais comme absolu en esprit et en vérité, qu'il passe de la sensibilité de la représentation à l'intériorité spirituelle », p. 141. Très souvent, dans l'Esthétique, Hegel, influencé par la théologie réformée, majore cette dimension de l'intériorité. S'il reconnaît par exemple le rôle du christianisme dans le développement des arts, il croit devoir constater un reflux à partir de la Réforme : « Lorsque l'impulsion vers la connaissance et la recherche ainsi que le besoin d'une spiritualité intime entraînèrent la Réforme, la représentation religieuse elle-même fut éloignée de l'élément sensible, et reconduite à l'intériorité de l'âme et de la pensée », p. 168. On peut naturellement prendre le contre-pied exact de cette dernière affirmation, dès lors qu'on interroge la spiritualité de la contre-Réforme tridentine, qui tend au contraire à rapprocher intériorité et " sensibilité ».

9. Dans le deuxième volet de La Gloire et la Croix, op. cit., Signes, l'auteur étudie, en diachronie, douze témoins de «l'esthétique théologique»: Irénée, Augustin, Denys, Anselme, Bonaventure, Dante, Jean de la Croix, Pascal, Hamann, Soloviev, Hopkins, Péguy. 
est censé lui conférer une autre orientation. Sans doute convient-il également, sur ce terrain piégé, de bien s'entendre sur la notion de Beau, trop unilatéralement référencée à Platon ou à Plotin. Avec habileté et prudence, Balthasar construit sa démonstration sur deux termes fondamentaux, indiscutablement validés par les Écritures : la gloire de Dieu (kabod, doxa, gloria) et la figure du Christ (tupos, character, figura), deux notions absolument centrales dans l'expression liturgique.

Dans la mesure où il cherche à saisir les reflets de la gloire divine sur la figure du Fils, gloire et figure déclinées ou représentées en leurs divers mystères, pendant l'année chrétienne, Nicolas Letourneux s'inscrit dans cette tradition de l'esthétique théologique, au-delà du seul sentiment religieux, mais non à l'exclusion de celui-ci. En commentant ce qui apparaît ou se révèle à partir de la Figure du Christ, au fil des offices, Letourneux développe une authentique mystagogie, au sens que Nicolas Cabasilas donnait à ce terme dans son Explication de la divine liturgie ${ }^{10}$. Nous voudrions justement sonder le processus d'une telle mystagogie à travers une sorte de cas limite, la liturgie du Vendredi Saint, rebelle a priori à toute approche esthétique, puisqu'en ce jour la Gloire s'obscurcit et que la Figure se défigure en s'abaissant jusqu'aux Enfers. « Si la croix, écrit Urs von Balthasar, est la fin radicale de toute esthétique terrestre, cette fin est précisément l'aurore décisive de l'esthétique divine $^{11}$. » On assisterait donc à une transmission de relais, à un passage, à une pâque de l'esthétique qui devrait à son tour, comme la Figure sacrifiée, renoncer à elle-même pour renaître métamorphosée. Le Vendredi Saint peut ainsi constituer l'épreuve majeure d'une telle démarche interprétative. Dans la mort-résurrection du Signe, en effet, on ne saurait diviniser la beauté, mais seulement expérimenter la beauté absolue et contradictoire de Dieu.

10. Explication de la divine liturgie, traduction et notes de Sévérien Salaville, Paris, Le Cerf, Sources chrétiennes $n^{\circ} 4$ bis (seconde édition), 1967. Nicolas Cabasilas, auteur byzantin du XIV siècle, voit dans l'accomplissement du sacrifice «l'œuvre essentielle de la mystagogie», p. 59, définie par ailleurs comme « le spectacle de cette divine économie »,p. 65. L'ouvrage de Cabasilas est cité à maintes reprises, comme témoin de la tradition liturgique, par Arnauld et Nicole, dans la Perpétuité de la foi catholique touchant l'eucharistie, et par Bossuet dans son Explication de quelques difficultés sur les prières de la messe à un nouveau catholique.

11. La Gloire et la Croix, op. cit., p. 389. 


\section{Paradoxe liturgiQue, Paradoxe esthétique}

En son dépouillement solennel, la liturgie du Vendredi Saint offre une physionomie singulière. On pourrait presque parler d'une liturgie sans liturgie puisqu'il s'agit du seul jour de deuil officiel dans l'Église, seul jour où la messe n'est pas célébrée. Obnubilé par l'événement commémoré, le sacrifice eucharistique est suspendu : « L'Église commence aujourd'hui l'Office qui tient lieu de Messe par l'exposition du Mystère dont elle célèbre la mémoire ${ }^{12}$. » Inversement, toutefois, on pourrait affirmer que ce Vendredi forme au contraire le seul jour vraiment liturgique, par une sorte d'antonomase hautement décisive : le Christ, seul leitourgos, exerce pleinement en ce jour son office public; le Serviteur accomplit son service en faveur du peuple. Sans Vendredi Saint, il ne saurait y avoir, à aucun autre moment, de liturgie authentique. C'est en ce sens que la dramaturgie de la messe se confond avec celle d'un Événement (la Passion) qui en révèle le sens véritable. La Cène, la Croix et le Sépulcre ne sont jamais considérés séparément et les différents moments du grand Shabbat scandent la liturgie du triduum pascal sans solution de continuité. L'Église omet la célébration du sacrifice non sanglant de l'Eucharistie parce que le Sacrifice sanglant de la Crucifixion constitue l'objet unique de la commémoration :

Aujourd'hui elle n'offre pas le sacrifice ordinaire, et elle se contente d'honorer le sacrifice sanglant que Jésus-Christ a offert une fois sur la Croix, et qui se continue tous les jours d'une manière non sanglante dans le sacrifice de la messe ${ }^{13}$.

La lecture de la Passion se substitue, par suppléance, à la messe, dont on a solennellement rappelé, la veille, l'institution, rappel

12. Année chrétienne..., op. cit., t. V, p. 334. Joseph de Voisin (1610-1685), aumônier du Prince de Conti, écrira de son côté : « On ne consacre point aujourd'hui parce qu'on célèbre seulement la mémoire de la Passion de Jésus-Christ selon qu'elle s'est passée actuellement », Messel [sic] romain, selon le règlement du Concile de Trente, traduit en français, avec l'explication de toutes les Messes et de leurs Cérémonies, pour tous les jours de l'année, t. III, Paris, Frédéric Léonard,

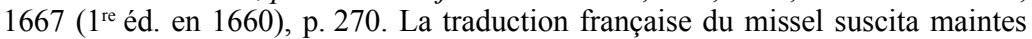
polémiques; une cabale, initiée par Mazarin, voulut faire croire que les portroyalistes souhaitaient une liturgie entièrement en français. Sur ces questions, voir, de Bernard Chédozeau, La Bible et la Liturgie en français, Paris, Le Cerf, 1990.

13. Année chrétienne... op. cit., p. 335. Voisin écrit: "L'oblation de ce jour n'est que la représentation du Sacrifice sanglant de Jésus-Christ », Messel... op. cit., p. 269-270. 
qui était déjà lui-même anticipation sacramentelle de la Passion. Comprendre le signe du Vendredi Saint revient d'abord à saisir la disposition des signes liturgiques mobilisés par une dramaturgie, et donc à comprendre justement le caractère éminemment dramatique de toute liturgie, en tant qu' « action sacrée » (hierourgia). Se pose alors un problème d'expressivité.

Comment représenter en effet un sacrifice sans la médiation du sacrement qui l'actualise? Le cadre habituel se modifie, puisque ce qui d'ordinaire précède ou accompagne la messe proprement dite (lectures, chants, prières) est ici amplifié au point d'envahir la quasitotalité de l'espace liturgique. Toute la célébration se confond avec la proclamation de la Parole, même si ce jour sans messe est précisément celui qui permet de comprendre le sens de toutes les autres messes célébrées dans l'année. Sans doute plus que les autres jours, la proclamation des Écritures vaut ici signe sacramentel. D'une certaine manière, le Vendredi Saint reste la clef sémiotique de tous les autres signes liturgiques, puisqu'en ce jour la Parole coïncide effectivement avec l'œuvre du salut.

Si la lecture du récit de la Passion devient «représentation » efficiente et spirituelle, ce spectacle se visualise dans l'audition et constitue un cas exemplaire du fides ex auditu. Letourneux avait déjà fortement mis l'accent sur cette réalité sensible et spirituelle dans De la meilleure manière d'entendre la sainte messe. L'Église, écrivait-il, « désire qu'on s'applique à ce qu'on voit, à ce qu'on entend, et à ce qu'on dit à la messe; elle souhaite qu'on la suive afin d'entrer dans les sentiments qu'elle tâche d'inspirer par les paroles qu'elle fait réciter ${ }^{14} »$. À la faveur d'une équivalence sensible (entre le voir et l'entendre), source d'émotions finalisées par le «sentiment» spirituel, un échange perceptif (synesthésie mystique) éduque le sens de la prière, c'est-à-dire aussi celui de la foi (lex orandi, lex credendi).

Paradigme explicatif de toutes les messes, en surimpression, la liturgie du Vendredi Saint organise la mise en scène d'un drame archétypal, la mort du Christ. C'est pourquoi, si Letourneux adopte dans son ouvrage une méthode qui consiste à offrir un écrin aux textes lus pendant l'année chrétienne, ici à plus forte raison se montre-t-il 
attentif à des textes étroitement liés aux rites qu'ils explicitent à la manière des didascalies théâtrales. Le commentateur met ainsi en valeur la structure hiératiquement responsoriale de l'Office : trois textes des Écritures entrecoupés de repons et de prières, longue prière d'intercession, double procession, celle de la Croix et celle des hosties consacrées la veille ${ }^{15}$. Au centre de cet ensemble dramatique, le récit johannique de la Passion est puissamment orchestré, traduit, incarné en des gestes ou en des chants qui en suggèrent une exégèse priante.

Or si l'on se tourne maintenant vers l'événement proprement dit, la mort du Christ, on constate qu'au paradoxe liturgique (la seule vraie liturgie mais sans la liturgie ordinaire) correspond un paradoxe esthétique, puisqu'il s'agit de célébrer un jour de deuil, sans éclat ni beauté apparente ${ }^{16}$. La figure du Christ (le «plus beau des enfants des hommes ${ }^{17}{ }^{17}$ ) se transforme en celle du Serviteur défiguré décrit par Isaï ${ }^{18}$. La liturgie ordonne textes et chants, ou bien codifie gestes et signes en s'efforçant de refléter la gloire divine, et cependant la Figure médiatrice n'est plus aujourd'hui que le Défiguré. Comment donc célébrer une défiguration, une difformité? N'y aurait-il pas, le cas échéant, une contradiction dans les termes? Cette difficulté, dans l'expression esthétique, confirme justement l'apparent non-lieu liturgique du Vendredi Saint, alors même, encore une fois, que ce jour promulgue la seule et unique liturgie chrétienne. Le paradoxe se rencontre dans le symbole expressif du noir liturgique. La couleur noire des ornements signifie sans doute le deuil, mais peut-être aussi l'envers ou le verso de toutes les autres couleurs liturgiques utilisées au cours du cycle annuel ${ }^{19}$. La mort du Christ fait précisément l'objet d'une couleur liturgique, comme si le spectre chromatique des autres couleurs trouvait son origine en ce noir fondateur (ténèbres premières du chaos primordial), qu'elles n'excluent jamais vraiment mais intègrent au contraire en profondeur.

15. Sur l'histoire du missel, consulter Histoire du missel français, Paris, Brepols, 1986, et surtout, de Joseph-André Jungmann, Missarum sollemnia. Explication génétique de la Messe romaine (3 vol.), Paris, Aubier, 1956.

16. Cf. Isaïe, le quatrième Chant du Serviteur, LIII, 2 : «sans beauté ni éclat pour attirer le regard $»$.

17. «Speciosus forma es prae filiis hominum », Psaume 45 (44), 3.

18. Isaïe, LII, 14 : « il n'avait plus figure humaine».

19. On sait que depuis Vatican II, l’Église catholique a cru devoir permettre la suppression des ornements noirs dans la liturgie afin de pas trop heurter la « sensibilité » contemporaine. 
Ainsi l'effacement de la pompe cérémonielle usuelle, aux accents triomphants, mime-t-elle le retrait d'une Gloire désormais enténébrée, alors même que cet obscurcissement provisoire révèle en sa plénitude le vrai sens caché de la Gloire divine. Tel est le paradoxe esthético-théologique de la liturgie pascale, et tel est sans doute le seul foyer nucléaire ou irradiant d'une possible esthétique théologique, qui ne peut se justifier, en effet, que dans le signe du Vendredi Saint. Comme le soleil sur le Golgotha, à l'heure du suprême abandon au Père, la Beauté s'est éclipsée, bien que son absence légitime dans l'invisible l'action liturgique accomplie (« tout est accompli »). La Croix porte jusqu'à l'incandescence mystique la Beauté inaccessible de Dieu, abaissée pourtant jusqu'à la mort. De ce point de vue, le Vendredi Saint est jour de révélation, jour d'apocalypse ${ }^{20}$. La défiguration du Serviteur implique un sacrifice esthétique de la liturgie, qui imite la kénose du Christ leitourgos, en empruntant à son tour la voie d'un retrait signifiant. Elle se condamne ainsi à la répétition d'une histoire, au commentaire lyrique, à la didascalie priante et méditative. Ces références à l'univers de la tragédie grecque ne doivent pas sembler anachroniques, ou trop chargés d'esthétique théâtrale : le christianisme primitif a souvent décrit le Christ comme un choreute, un aède, un mélode venu en ce monde apporter le chant nouveau ${ }^{21}$. En ce jour d'ailleurs, l'Église latine, comme l'Église byzantine, a toujours pris soin de cultiver une sorte de conservatisme liturgique, par le maintien des formes les plus archaïques du chant sacré ou de la psalmodie ${ }^{22}$.

20. Le livre de l'Apocalypse de saint Jean, saturé de symboles, est comme rythmé par les différents épisodes d'une liturgie céleste, exégèse imagée du Vendredi Saint et du mystère pascal.

21. Hippolyte de Rome, par exemple, au $\mathrm{III}^{\mathrm{e}}$ siècle, désigne le Christ comme " le coryphée de la danse mystique », cité dans Prières des premiers chrétiens, A. Hamman, Paris, Fayard, 1952, p. 69. Clément d'Alexandrie use d'un lyrisme semblable.

22. Sur la manière dont le XVII ${ }^{\mathrm{e}}$ siècle concevait la psalmodie et la nature de la musique sacrée, voir, du cardinal Bona, De divina psalmodia ejusque causis, mysteriis et disciplinis, Deque variis ritibus omnium Ecclesiarum in psallendis divinis officiis, Tractatus historicus, symbolicus, asceticus, sive psallentis ecclesiae harmonia, Paris, Louis Billaine, 1663. On lit par exemple, au chapitre 17, De cantu ecclesiastico : "Nullum est tam immite, tam asperum pectus, quod non musicis sonis capiatur [...] O immensam musicae suavitatis efficaciam! Quam dulci tyrannide in omnes microcosmi potentias dominaris!», p. 407. En un style aussi marqué par l'humanisme, le pape Urbain VIII avait publié en 1631 la bulle Divinam psalmodiam. 
Dans ses commentaires, Letourneux imite lui aussi la grave discrétion de la liturgie en insistant sur « l'exposition du mystère » qui remplit l'Office du jour ${ }^{23}$. Sur la Croix, le Verbe s'efface quand meurt l'hypostase du Fils, et cet effacement se reproduit à nouveau lors de la proclamation de la Passion. Plus que jamais il convenait, pour Letourneux, de faire parler les textes afin d'en mieux déployer le sens.

\section{EXPOSITION, DISPOSITION, DÉPOSITION}

L'Année chrétienne ne relève aucunement de la paraliturgie; elle ne se substitue jamais en effet à la liturgie, qu'elle s'efforce seulement de faire parler ${ }^{24}$. D'autre part, si l'objectif principal consiste à « inspirer des sentiments » pour mieux soutenir l'attention pendant l'assistance aux offices (ce par quoi l'ouvrage dévoile implicitement un arrière-plan esthétique), il ne peut s'agir en aucune manière de favoriser un quelconque sentimentalisme dévot. La notion de désir spirituel, sur laquelle Letourneux insiste tant, dans De la meilleure manière d'entendre la sainte messe, se réfère exclusivement à la dimension ecclésiale de la liturgie :

Mais à quoi penser, et que désirer dans la messe? C'est ce que l'Église apprend aux fidèles par tout ce qui s'y dit, et par les cérémonies mêmes dont elle se sert dans la célébration des mystères ${ }^{25}$.

Alors que se répandent, au XVII ${ }^{\mathrm{e}}$ siècle, différentes " méthodes" pour favoriser une meilleure participation à la messe (très souvent réduites à la récitation de prières privées sans grand rapport avec l'action liturgique, en dépit d'une recommandation pressante du Concile de Trente), Letourneux soutient que la messe génère sa propre « méthode ». L'Église seule, dans la liturgie, oriente le désir des fidèles

23. Les Matines du Vendredi Saint sont intensément et puissamment lyriques. Sur la constitution progressive du Bréviaire, voir, de Pierre Batiffol, Histoire du bréviaire romain, Paris, Picard, 1893. Sur l'élaboration des Lectionnaires liturgiques, consulter, de A.G. Martimort, Les Lectures liturgiques et leurs livres, Typologie des sources du Moyen Âge occidental n 64, Turnhout, Brepols, 1992.

24. Sainte-Beuve a très bien perçu le caractère mystagogique de l'Année chrétienne: «On se rend compte, si l'on est croyant, des motifs de sa foi et de son culte, des diverses formes et des appropriations de la prière, de la composition et de l'ordonnance que l'Église a données à l'Année chrétienne, et de l'appui qu'y trouve une âme chrétienne à chaque instant, de la station qu'elle y peut faire à chaque degré », Port-Royal, op. cit., p. 232.

25. De la meilleure manière..., op. cit., p. 193-194. 
par le biais des signes sensibles. De même qu'il convient de s'appliquer « à ce qu'on voit » et «à ce qu'on entend », il faut s'imprégner du spectacle liturgique pour désirer ce que l'Église désire :

Celui qui s'occupe l'esprit et le cœur des paroles et des actions qui composent la liturgie se conforme infailliblement davantage à l'esprit et aux sentiments de l'Église que celui qui s'occupe de tout autre objet, quelque saint qu'il puisse être ${ }^{26}$.

Malgré sa complexité sémantique, le mot «sentiment» ne doit pas être compris dans son acception romantique. Ancré dans l'étymologie, le mot renvoie conjointement à l'expérience physique et spirituelle des sens, à une étroite analogie entre perception et compréhension, à un secret isomorphisme des sens corporels et des intuitions intellectuelles. Or le spectacle liturgique travaille puissamment à cette symbiose qui nourrit le vrai désir spirituel. Par la déclinaison de son radical, en ses nombreux dérivés, le terme « sentiment » suppose la sensation corporelle, l'assentiment de l'esprit, le consentement du cœur. D'une certaine manière, Letourneux donne un relief saisissant, dans un cadre liturgique, à la célèbre formule ignacienne : sentire cum ecclesia. Mais ici il ne s'agit que de liturgie, et donc d'une expérience physique et spirituelle du Mystère. "Suivre » la messe consiste à sentir ou à ressentir ce que toute l'Église, à travers les siècles, a vécu dans son corps, dont le Christ est la Tête. « Suivre » enfin les offices du Vendredi Saint, qui explique toute messe, revient à emprunter, au physique comme au spirituel, la via dolorosa tracée par le Serviteur, itinéraire historique certes (trajet qui conduit au Calvaire) mais surtout chemin d'abaissement du Sauveur, depuis sa naissance jusqu'à sa mort. La procession de la Croix, après la lecture de la Passion, signifie cette participation concrète des fidèles, qui n'assistent pas au spectacle justement en tant que spectateurs passifs, mais en qualité de membres du Corps souffrant et glorifié. Acteurs de la divine dramaturgie, ils se font servants avec le Serviteur.

Dans une telle perspective on comprend davantage l'expression programmatique employée par Letourneux au début de son commentaire du Vendredi Saint : « honorer le sacrifice ${ }^{27}$ ». Rites et paroles ne cessent jamais d'être doxologiques, dans un processus liturgique de glorification. La louange doxologique traverse toute «l'expo-

26. De la meilleure manière..., op. cit., p. 194.

27. Année chrétienne, op. cit., p. 335. 
sition du mystère ${ }^{28} »$, c'est-à-dire la disposition des différentes actions liturgiques. Comme Joseph de Voisin dans sa traduction du missel, Letourneux se montre attentif à rendre intelligible la cohérence d'une expression (succession des rites, rapports qu'ils entretiennent). La notion rhétorique de dispositio se traduit en théologie par celle d'économie, et ce sont l'une et l'autre de ces notions qui gouvernent l'ordonnance des cérémonies. Nicolas Cabasilas faisait lui-même très souvent mention de cet ordre (taxis) signifiant ${ }^{29}$. Si la disposition liturgique est nécessairement porteuse de vérité (par son contenu doctrinal) et de charité (par diffusion de la grâce), elle ne peut être que belle en raison du rayonnement de la gloire qui émane de la mort-résurrection du Christ. Le troisième des transcendantaux, autrement dit la dimension esthétique, ne saurait être exclu, car ne pas être « sensible » à la Beauté signifierait ne pas discerner la gloire de Dieu. Puisque le projet de l'Année chrétienne repose sur l'arrangement formel, dans la syntaxe liturgique, de l'économie rédemptrice, le regard porté sur cette disposition est en quelque sorte dès le départ un témoignage sur l'esthétique théologique qui le justifie ${ }^{30}$.

Letourneux contemple, dans les lectures du Vendredi Saint, le déploiement d'une économie salvifique que Joseph de Voisin pour sa part s'efforce de transcrire à partir du symbolisme des gestes rituels $^{31}$. Dans les deux cas s'exprime à leurs yeux la signifiance secrète de ce que Cabasilas appelait «l'harmonie »:

La mystagogie tout entière est comme une représentation unique d'un seul corps (eikon mia enos somatos), qui est la vie du Sauveur;

28. Année chrétienne, op. cit., p. 334.

29. Cabasilas établit une analogie entre le «bon ordre des divins mystères (ten taxin tôn musteriôn) » et "l'arrangement du rite sacré (tè suntaxei tès hieras teletès) ». Cela nous « dispose soit à bien recevoir, soit à bien conserver la sanctification », Explication de la divine liturgie, op. cit., p. 58-59. Le mot taxis renvoie en outre au verset 4 du psaume $110(109)$ : «Tu es prêtre à jamais selon l'ordre de Melchisédech », verset cité à maintes reprises par Hébreux, V-VII.

30. La même dimension esthétique, c'est-à-dire esthético-théologique, se rencontre dans cette remarque de Pascal sur l'arrangement ou la disposition des événements de l'histoire (taxis et syntaxis encore, mais cette fois dans l'ordre de la Providence) : «Il est beau de voir par les yeux de la foi Darius et Cyrus, Alexandre, les Romains, Pompée et Hérode agir sans le savoir pour la gloire de l'Evangile! », Pensées, éd. Sellier, fr. 348.

31. Par exemple : une seule nappe sur l'autel, pour signifier le linceul, Messel, op. cit., p. 223; lecture de la Passion sur un pupitre nu, pour rappeler la nudité du Christ, p. 234; point d'encensement, afin de marquer le refroidissement de la foi des disciples, p. 246, etc. 
elle met sous les yeux les diverses parties de cette vie, du commencement à la fin, selon leur ordre et leur harmonie ${ }^{32}$.

Le souci de rattacher le moindre détail symbolique au mystère d'une économie communiquée sensiblement par des signes montre combien le XVII ${ }^{\mathrm{e}}$ siècle, animé par son zèle pastoral, n'a voulu abandonner aucun symbole liturgique à la seule discipline formelle du culte. Le même refus de ce qui pourrait n'être qu'un rubricisme stérile se retrouve d'ailleurs dans le Thesaurus de Bartholomeo Gavanto, commentaire magistral des rubriques du missel et du bréviaire $^{33}$. Comprises comme des didascalies, les rubriques du missel, pour De Voisin comme pour Letourneux, soulignent par leur sobre délicatesse toute la beauté contemplative des cérémonies.

L'ordonnance des gestes reflète l'ordonnance des textes, avec la même puissance sensible, tandis que «l'exposition du mystère » épouse la disposition liturgique ${ }^{34}$. L'exposé de Letourneux se conforme à ce que Cabasilas décrivait comme « représentation christique (ton Christon tupomenon) ${ }^{35} »$, non sans faire porter l'accent sur l'indispensable association du corps à la perception du mystère :

Voici pourquoi a été imaginé le symbolisme (tupos) dont j'ai parlé, qui ne se borne pas à signifier tout cela par des paroles, mais le met entièrement sous nos yeux, et cela visiblement à travers tout le cours de la liturgie : c'est, d'une part, pour agir plus facilement sur nos âmes, ne pas nous offrir une simple vision, mais encore déposer en nous un sentiment (pathos), car une représentation visuelle peut produire en nous une impression plus profonde ${ }^{36}$.

Letourneux n'emploie pas le mot « sentiment» dans un sens différent, et il « expose » le mystère comme « la représentation unique d'un seul corps », « comme un seul corps de récit ${ }^{37} »$. Au fil de

32. Explication de la divine liturgie, op. cit., p. 62-63.

33. Thesaurus sacrorum rituum seu commentaria in rubricas missalis et breviarii romani, Antverpiae, ex officina Plantiniana Balthasaris Moreti, 1634.

34. Le terme « exposition » employé par Letourneux rappelle celui d'anadeixis, utilisé par Cabasilas avec le sens de " geste qui montre », " ostension ». La saveur liturgique du terme se retrouve particulièrement dans la pratique tridentine de l'exposition du Saint-Sacrement dans l'ostensoir.

35. Cabasilas écrit « dans ces formules et ces rites nous voyons la représentation du Christ », Explication de la divine liturgie, op. cit., p. 61; ici le traducteur traduit par représentation le mot tupomenon; plus loin il traduit également par représentation le terme eikon (image), p. 63.

36. Explication de la divine liturgie, op. cit., p. 69.

37. Explication de la divine liturgie, op. cit., respectivement p. 63 et 129. 
l'Année chrétienne, exposer signifie poser et proposer, déployer pour montrer. Letourneux ne paraphrase ni ne spiritualise à outrance (procédés assez courants dans la littérature dévote du grand siècle), mais il étire ou plutôt « représente » le sens, c'est-à-dire qu'il explique véritablement. Ainsi retrace-t-il la fresque de la Passion, déjà contenue en abrégé ou en figures dans les textes prophétiques, sans accorder aux textes d'Osée ou de l'Exode ${ }^{38}$ moins d'importance matérielle qu'au récit johannique de la Passion. Le commentateur s'attache à montrer non seulement l'accomplissement des figures mais surtout la révélation définitive, cruciale, de l'unique Figure, défigurée et exaltée, Figure actualisée dans l'hodie de la liturgie. En effet, au jour de la plus incompréhensible des théophanies (le Crucifié), la Figure fait cesser, une fois pour toutes, le suspense figuratif entretenu par les prophètes. Ce processus laisse aisément transparaître sa dimension esthétique puisqu'il implique une épiphanie de la gloire à travers une figure singulière. Il devient un processus de révélation par lequel transitent ou apparaissent les contours circonscrits de l'hypostase christique, abîmée jusqu'au néant au terme de son parcours mortel ${ }^{39}$.

En réalité, Letourneux comprend le Vendredi Saint, non comme un dévoilement mais comme le dévoilement par excellence de la Gloire. Quand les ténèbres recouvrent une terre qui tremble ${ }^{40}$, la nuée pourtant se dissipe et l'Arche d'Alliance paraît à découvert. La Croix devient le vrai Buisson ardent où se révèle l'intimité confidentielle de Dieu. «Suivre» l'office de ce jour permet de saisir quelque peu cette aveuglante splendeur, avec l'émotion et le désir, dans la foi et la charité, au terme d'un lent processus de « reconnaissance », comme disait Aristote à propos du dénouement tragique ${ }^{41}$. Alors les perspectives s'inversent, dès lors qu'au moment où la gloire semblait exilée, elle atteint au contraire sa plénitude secrète,

38. Osée, VI (annonce de la résurrection « le troisième jour ») et Exode, XII (sur la manière de manger l'agneau pascal).

39. Sur l'importance de la notion de «circonscription" dans la théologie de l'icône, consulter l'ouvrage remarquablement clair de Christoph Schönborn, L'icône du Christ. Fondements théologiques, Paris, Cerf, 2003 (1 $1^{\mathrm{re}}$ éd. Fribourg, 1976)

40. Ces manifestations naturelles ou cosmiques sont mentionnées dans Matthieu, XXVII, 45 et 51. Différents repons de l'Office des Matines du Vendredi Saint répercutent l'écho lyrique de ces événements prodigieux.

41. De fait, tout le récit de la Passion selon saint Marc, par exemple, se termine par un acte d'humble « reconnaissance» posé par un centurion anonyme : «Vraiment cet homme était fils de Dieu », Marc, XV, 39. 
dans la « folie de la croix ». Et puisque la chair du Christ (reçue, meurtrie, offerte) coïncide ici avec l'Esprit qu'il rend à son Père, en un échange inouï, les mots de l'Écriture atteignent eux aussi leur complet achèvement. Quand la Figure meurt et se transfigure, sens littéral et sens spirituel ne sont plus discriminés entre eux. L'affirmation sans doute la plus éclairante de Letourneux, sous cet aspect, réside dans son commentaire d'Osée :

Ce langage figuré pour signifier que l'affliction d'Israël ne durera pas longtemps [...] n'est plus figuré mais littéral, pour signifier le grand Mystère que nous révérons en ce jour ${ }^{42}$.

Sous les yeux en effet, pendant la « représentation » liturgique, ne subsiste que la figure énigmatique du Crucifié, dont la vérité échappe à l'épreuve des sens et qui ne se soustrait au regard que pour resurgir dans la nuit du surlendemain. Au fidèle qui assiste à la liturgie du Vendredi Saint, une expérience d'illumination reste offerte, au-delà d'une simple compréhension d'ordre intellectuel, au-delà également d'un banal sentiment de compassion ou d'un pur choc émotionnel, même motivé par la plus fervente piété. L'expérience est bouleversante, au plus profond de l'être, qui révèle l'irruption d'une Transcendance absolue et laisse deviner, intuitivement, l'existence d'une beauté hors normes provenant de Dieu et en lui se perdant. Saint Augustin avait raconté une semblable expérience de conversion du regard en des termes déjà hardiment esthétiques : «Bien tard je t'ai aimée, ô beauté si ancienne et si nouvelle ${ }^{43}$. » $\mathrm{Au}$ fond, Letourneux place le fidèle devant le fait accompli. « $\mathrm{Ce}$ langage figuré... n'est plus figuré » : toute la liturgie du Vendredi Saint mime symboliquement la déchirure du Voile ${ }^{44}$.

L'erreur consisterait à s'imaginer qu'en pareilles circonstances l'esprit seul est sollicité, au détriment du corps, ce qui serait pourtant contraire à la logique de l'Incarnation. Le corps demeure toujours « temple de l'Esprit » et la chair « cardo salutis », comme le disait Tertullien. Parce qu'elle est mystagogie, la liturgie se transmue en

42. Année chrétienne, op. cit., p. 337.

43. Confessions, Bibliothèque augustinienne, Paris, Desclée de Brouwer, tome 2, 1962, Livre X, p. 209. On connaît par ailleurs l'abondance du lexique sensible et sensuel chez Augustin, en particulier dans les Confessions : lire par exemple l'utilisation contemplative des cinq sens, Livre X, 6, p. 153-155.

44. "Velum templi scissum est», Matthieu, XXVII, 51. L'expression est reprise dans l'un des repons des Matines du Vendredi Saint. 
signe (dans le sens que le Nouveau Testament donne à ce terme : image, type, figure ${ }^{45}$ ) et ce signe convoque une interprétation, une appropriation, une incorporation. Letourneux réaffirme nettement la fonction de relais joué par le signe, qui n'est validé, sur le plan liturgique, que s'il est assumé dans sa matérialité et ensuite converti en disposition intérieure :

Tout le culte extérieur de la religion ne doit être que le signe du culte intérieur que l'homme doit à celui qui étant esprit et vérité, demande, comme Jésus-Christ nous l'enseigne, des adorateurs en esprit et en véritét $^{46}$.

Letourneux écrit ces mots pour justifier la vénération sensible du bois de la Croix, cérémonie qui répond au récit de la Passion, par son lyrisme gestuel, à la manière de ces antistrophes chantées par le Chœur dans la tragédie grecque ${ }^{47}$. En dépit des caricatures, en effet, le XVII ${ }^{\mathrm{e}}$ siècle ne s'est montré nullement réfractaire à ce que Bossuet lui-même nommait «le langage du corps ». Dans ses écrits de controverse à l'encontre des ministres réformés, Bossuet défend la signification spirituelle des « actes extérieurs », « celui de la parole » et « celui de tout le corps » :

Ces deux sortes de culte extérieur ont une grande affinité. Car les génuflexions et autres actions de cette nature, après tout, ne sont autre chose qu'un langage de tout le corps, par lequel nous expliquons, de même que par la parole, ce que nous sentons dans le cœur ${ }^{48}$.

Pendant qu'on vénère la Croix, une médiation sensible, incarnée par des gestes concrets, opère un transfert de la parole à l'icône, pour finalement redire la même chose ou retracer la même histoire.

45. Ainsi par exemple, $L u c$, II, 12, à l'occasion de la naissance de Jésus : « Et cela vous servira de signe (semeion). »

46. Année chrétienne, op. cit., p. 341.

47. Une tragédie grecque, sur la passion du Christ, composée d'une mosaïque de textes empruntés aux Ecritures et à Euripide, a souvent été attribuée à saint Grégoire de Nazianze, avec un fort degré de certitude d'après son dernier éditeur, André Tuilier : La Passion du Christ, Paris, Le Cerf, Sources chrétiennes n 149, 1969.

48. Du culte dû à Dieu, Euvres de Bossuet, Paris, Mequignon, tome huitième, 1846, p. 7. Bossuet ajoute : «Les actions extérieures de respect, que nous avons appelées le langage de tout le corps, s'accordent avec le langage de la voix. On ne prétend expliquer, par ces actions, que la même chose qu'on dit, et l'un de ces langages doit être entendu par l'autre, de sorte que si l'un est bon, on ne doit pas présumer que l'autre soit mauvais », p. 8. On notera l'équivalence sémiotique, dans la liturgie, entre geste et parole. On peut s'étonner aussi, de manière plus anodine, de la naïveté avec laquelle le $\mathrm{xx}^{\mathrm{e}}$ siècle a cru avoir « inventé » le concept de « langage du corps ». 
Chacun des participants (clergé, laïcs) est appelé à s'engager physiquement, à exprimer son adoration en un « langage » qui lui propre. Pour éviter que le geste soit vide, par dérive formaliste, Letourneux précise qu'il n'est que « le signe du culte intérieur », en somme un simple vecteur spirituel. Si l'extérieur ne se confondait pas avec l'intérieur, la célébration ne serait qu'apparence, piégée par la seule esthétisation des signes. Or le signe liturgique reflète le rayonnement de la Figure christique, selon les paroles de saint Paul, « pour faire briller la connaissance de la gloire de Dieu, qui est sur la face du Christ $\gg{ }^{49}$. Le visage du Christ, même bafoué, conserve un éclat divin. Au cours du Vendredi Saint, un lien rituel rythme une double transition du signe : la proclamation de la Passion se réitère autrement dans la vénération processionnelle de la Croix, elle-même suivie par une autre procession, celle qui ramène à l'autel l'hostie consacrée la veille. Cette double procession, par la médiation du signe (bois de la croix, pain sanctifié) actualise le mouvement d'incarnation du Verbe. Si le mystère est «exposé » dans la Parole, il est réexposé ensuite à deux reprises à travers le double mouvement processionnel. La disposition dramaturgique de ces différentes actions s'achève alors par la communion au Corps déposé sur l'autel.

\section{Trône VIDE, SIÈGE VACANT}

Trois lieux sont ainsi traversés par la divine Présence : la parole, l'icône et le sacrement ${ }^{50}$. En ce jour du Vendredi Saint - le seul de l'année où, dans l'église latine, une icône joue un rôle essentiel dans le déroulement liturgique -, apparaît fortement le lien qui unit l'icône ${ }^{51}$, où l'hypostase est figurée sans la substance, au sacre-

49. II Corinthiens, IV, 6.

50. Bossuet a écrit tout un opuscule Sur l'adoration de la Croix (adressé à un protestant converti); il lit dans la Croix un message resplendissant, celui du Vendredi Saint : « Tout l'appareil de ce jour-là ne tend qu'à faire sentir aux fidèles les merveilles de la mort de Jésus-Christ; l'Église les ramasse toutes en montrant la Croix, où, comme dans un langage abrégé, elle nous dit tout ce que le Sauveur a fait pour nous : on les voit toutes dans ce seul signal, et comme d'un coup d'œil », Euvres, op. cit., p. 471, c'est nous qui soulignons l'arrière-plan esthétique des formules.

51. On se rappellera que le Concile de Nicée II (en 787) appuie sa justification cultuelle des saintes images sur l'analogie relative qu'elles entretiennent avec « la représentation (tupos) de la précieuse et vivifiante croix », ainsi conçue comme le 
ment qui, à l'inverse, contient la substance mais sans l'hypostase. À chaque fois, et bien que selon des modalités différentes, se trouve communiquée la gloire paradoxale du Crucifié.

Les hymnes chantées en ce jour témoignent de cette doxologie déconcertante : au très antique Trisagion (chanté en grec et en latin $)^{52}$ succèdent deux chants aux accents de victoire, le Pange lingua ... et le Vexilla regis ${ }^{53}$. Dans sa version française des hymnes, Letourneux se permet une traduction glosée qui renforce le paradoxe doxologique du mystère de la Passion : d'une part il traduit l'expression « triumphum nobilem » par « triomphe éclatant ${ }^{54} »$, en survalorisant la dimension esthétique de la louange, d'autre part il explicite et souligne le scandale du Dieu anéanti en traduisant «temps de la passion » par « temps sacré des souffrances d'un Dieu ${ }^{55} »$. En conciliant ces deux extrêmes (le plus grand éclat dans l'abaissement kénotique), Letourneux contemple une défiguration où se cristallise un admirabile commercium. La beauté peut alors jaillir et rejaillir, bien que mystérieusement enfouie dans l'invisible et l'inacceptable. Commenter la liturgie du Vendredi Saint implique le déchiffrement du Signe à l'instant même où son apparition coïncide avec son énig-

modèle théorique de toute icône, Les Conciles æecuméniques, Les décrets, t. II-1, Paris, Le Cerf, 1994, p. 135-136.

52. Déjà en usage à Constantinople au IV ${ }^{\mathrm{e}}$ siècle, ce chant est passé tel quel dans la liturgie romaine.

53. Dom Guéranger souligne la finition esthétique de ces hymnes : «Les chants qui accompagnent l'adoration de la Croix sont de la plus grande beauté »; à propos du Trisagion, il note que ce chant « est du plus haut intérêt dramatique », L'Année liturgique, Poitiers, Henri Oudin et Paris, Victor Palmé, 1869, La Passion et la Semaine Sainte, p. 553.

54. Il s'agit de la première strophe du Pange lingua... (hymne composée par Mamert Claudien au $\mathrm{VI}^{\mathrm{e}}$ siècle) : "Pange lingua gloriosi/Lauream certaminis/ Et super Crucis tropaeum/Dic triumphum nobilem/Qualiter redemptor orbis/ Immolatus vicerit. » Letourneux traduit: "Que notre langue chante le glorieux combat que Jésus-Christ a soutenu sur la Croix, et le triomphe éclatant qu'il y a remporté; qu'elle dise comment le rédempteur du monde a vaincu en s'immolant comme une victime », Année chrétienne, op. cit., p. 329. Le Pange lingua... est chanté pendant la cérémonie de la vénération de la Croix.

55. Ici, il s'agit de la dernière strophe du Vexilla regis... (hymne composée par Venance Fortunat au $\mathrm{vl}^{\mathrm{e}}$ siècle) : «O Crux, ave, spes unica/Hoc passionis tempore/ Auge piis justitiam/Reisque dona veniam. » Letourneux traduit ainsi : "Nous te vénérons, ô Croix, notre unique espérance. Que par toi dans ce temps sacré des souffrances d'un Dieu, les justes croissent en piété, et que les pécheurs obtiennent le pardon de leurs crimes ", Année chrétienne, op. cit., p. 332. Le Vexilla regis... est chanté pendant la procession qui ramène le Saint-Sacrement à l'autel, depuis le Reposoir. 
matique disparition. Aux yeux de la foi, l'expression « croix glorieuse » ne constitue pas un oxymore mais un pléonasme.

Ainsi, à l'instar de la liturgie officielle, Letourneux n'a de cesse de montrer une œuvre de glorification, et cela pour deux raisons. La première est d'ordre dogmatique, avec la réaffirmation très claire du mystère christologique (contre toutes les déviations résurgentes de l'arianisme, du docétisme, du gnosticisme); la seconde raison relève plus immédiatement du domaine spirituel, avec une sorte de mise en garde tacite contre toutes les formes de sentimentalisme trompeur qui aboutiraient à confondre émotion psychologique et acte de foi. Malgré l'évidente nécessité d'éveiller des « sentiments » (Cabasilas parlait de pathos), ni la liturgie ni Letourneux ne cèdent à la phraséologie sentimentale, et encore moins à un esthétisme réducteur, simpliste et déplacé ${ }^{56}$.

De quel type de révélation est-il question en effet, même sur le plan de l'esthétique théologique? Il s'agit de montrer une transfiguration qui opère dans l'imperceptible et l'inintelligible, de croire en la métamorphose secrète des apparences, changement à vrai dire bien plus incroyable que celui de la Transfiguration sur le Thabor, mais non moins glorieux, ou plutôt bien plus glorieux que celuici. Une telle métamorphose $\mathrm{e}^{57}$ renvoie à la metabole eucharistique, changement substantiel sous l'apparence du signe ${ }^{58}$. Si donc la

56. On ne peut pas, ici, ne pas invoquer Chateaubriand qui résume la semaine sainte par une accumulation désordonnée de détails ou de symboles entièrement détachés de leur portée liturgique : «Qui pourrait représenter ce clergé en deuil, ces autels, ces temples voilés, ces cloches muettes, cette musique sublime, ces voix célestes chantant les douleurs de Jérémie, cette passion mêlée des plus incompréhensibles mystères, ce saint sépulcre environné d'un peuple abattu, ce pontife lavant les pieds des pauvres, ces épaisses ténèbres, ces silences entrecoupés de bruits formidables, enfin ce cri de victoire échappé tout à cour du tombeau, ce Dieu triomphant, qui ouvre la route du Ciel aux âmes délivrées, et qui laisse au chrétien vertueux sur la terre, avec une religion divine, d'intarissables espérances », Génie du christianisme, t. VII, Lyon, Ballanche, 1804 (4éd.), p. 63-64. Urs von Balthasar voit en Chateaubriand un « apologiste esthétique », un exemple en quelque sorte de la théologie esthétique, si opposée à l'esthétique théologique, La Gloire et la Croix, op. cit., p. 75. Il parle de son « mauvais goût », p. 77, et sans doute peut-on trouver ce pathos romantique assez désastreux ou consternant sur le plan liturgique et spirituel.

57. C'est le mot même employé par le texte des Évangiles; voir par exemple Marc, IX, 2 (metemorphote).

58. Nicolas Cabasilas commence son ouvrage par ce constat : «Dans la célébration des saints mystères, l'acte essentiel est la transformation (metabole) des dons offerts qui deviennent le corps et le sang divins », Explication de la divine liturgie, op. cit., p. 57. On sait que l'Eglise catholique parle de « transsubstantiation ». 
Figure est incompréhensible sans la transfiguration qu'elle porte en elle, le signe de la Croix devient l'ultime signature de la gloire. En un «triomphe éclatant» culmine la manifestation de la vérité, vrai désir du peuple fidèle. La question que Letourneux posait luimême (" que désirer dans la messe ? ») trouve un autre élément de réponse dans son commentaire de la célèbre question formulée par Pilate : « Pilate, qui prit apparemment Jésus-Christ pour un philosophe, lui demanda : qu'est-ce que la vérité, mais avec peu de désir de l'apprendre ${ }^{59}$. » Objet de désir, la vérité ne relève ni du voir ni du savoir; elle s'insinue dans le cœur, s'impose dans l'effacement, rayonne au sein de l'obscurité et illumine par sa splendeur : «On devient vraiment libre par cette soumission à la vérité qui rend rois tous ceux qui se laissent conduire par elle ${ }^{60}$. » Le chrétien est luimême transfiguré par sa configuration au Christ.

Letourneux donne un relief saisissant à ce dynamisme liturgique du Vendredi Saint ${ }^{61}$. Commentant les prescriptions de l'Exode à propos du repas pascal, il écrit :

Il faut manger ce divin agneau comme des voyageurs. C'est une victime de passage, et que nous ne devons manger qu'en passant. Il n'y aura plus d'eucharistie au Ciel où nous serons dans notre pays ${ }^{62}$.

59. Année chrétienne, op. cit., p. 360.

60. Année chrétienne, op. cit., p. 361. Dans une prière finale, qu'il place au terme de son commentaire, Letourneux revient une dernière fois sur l'équivalence (chère aux poètes mystiques) entre ténèbres et lumière : "Que la vérité persécutée et crucifiée ne soit pas moins l'objet de notre adoration que la vérité glorieuse et triomphante ", Année chrétienne, op. cit., p. 373.

61. Soucieux de défendre Letourneux contre les attaques de Dom Guéranger (qui ne supportait pas son "gallicanisme » et qui le critique sévèrement dans les Institutions liturgiques), Bremond admire son œuvre d'apôtre de la liturgie (il l'appelle même, avec ironie mais à juste titre, «le Dom Guéranger de ce tempslà ", Histoire littéraire ..., op. cit., p. 38). Cela dit, Bremond ne cite quasiment pas L'Année chrétienne et reste victime, quoi qu'il en dise, des préjugés de son temps, notamment en ce qui concerne l'École de Port-Royal. D'où, par exemple, ce jugement assez conformiste, qui ne semble pas avoir sondé la spiritualité liturgique de Letourneux : «Rigide plus que rigoriste, grave plutôt que triste. La passion moralisante et l'incompréhensible sérieux du grand siècle. C'est la marque du classicisme dévot. Mais, si curieux que cela nous semble, cette morne littérature les mettait en dévotion. Ai-je besoin d'avertir que Nicolas Letourneux n'avait pas lu le Génie du christianisme. Moins fermé néanmoins que nous ne pourrions le croire à la splendeur de nos offices. Pas besoin non plus de répéter que je préfère, et de beaucoup, les carillons de Dom Guéranger à la crécelle de Letourneux », p. 48. Étonnant verdict qui semble considérer que la « splendeur » liturgique n'a été perçue qu'à partir de Chateaubriand !

62. Année chrétienne, op. cit., p. 350-351. 
La vie chrétienne suit le rythme pressant de ce mouvement migratoire: «On va à Dieu par les désirs, on s'approche de lui par les bonnes œuvres, on y arrive par la charité ${ }^{63}$. Toute la liturgie de la Passion exemplifie cette série de passages effectifs, cette séquence de pâques successives : le corps du Christ passe à travers la parole proclamée, transite dans l'icône et se substitue au pain; il est constamment en procession, nomade jusqu'au tombeau où il ne réside que pour en sortir «sous une autre forme ${ }^{64} »$. Exposé aux yeux des foules, déposé de la Croix pour enfin reposer dans le sépulcre, il dérobe sa propre beauté, sa figure de gloire, assurant par là même le triomphe du Signe en son renoncement suprême, dans le néant de toute signification raisonnable ${ }^{65}$.

La plus inconcevable des beautés n'a pas à se justifier; elle est, tout simplement, en un retrait surnaturel, et elle suppose, chez celui qui la contemple, une série de ruptures sans condition (psychologiques, sociales, culturelles, philosophiques, spirituelles). Dans le texte même des Évangiles nombreux sont les indices ou les symboles de cette brisure décisive qui enfante un monde nouveau : voile déchiré, côté transpercé, tombeau fracturé. Antiquum documentum novo cedat ritui, écrivait saint Thomas d'Aquin ${ }^{66}$ : cette transmission est tout à la fois rétrocession cultuelle (du passé vers l'avenir), tradition d'alliance et testament définitif. La fin de l'Office, avec son annihilation totale des signes sensibles, n'expose plus qu'un trône vide, un siège vacant, une icône effacée. Toute la liturgie du Vendredi Saint aboutit à cette étimasie ${ }^{67}$, quand l'absence irréelle de Dieu signifie, sous l'effet d'une mystique rétroversion - œuvre de grâce - une présence plus réelle que la réalité elle-même.

32, impasse des Aubépines

34830 Jacou

christian_belin@hotmail.com

63. Année chrétienne, op. cit., p. 351.

64. "En hetera mophe (in alia effigie) », Marc, XVI, 12.

65. Voir, de Urs von Balthasar, Leben aus dem Tod, Einsiedeln, Johannes Verlag, 1997.

66. Hymne Pange, lingua, gloriosi..., pour le Saint-Sacrement, str. 5.

67. C'est l'un des grands thèmes de l'iconographie byzantine. 